\title{
Scattering equations and virtuous kinematic numerators and dual-trace functions
}

\author{
Stephen G. Naculich ${ }^{1}$ \\ Department of Physics, Bowdoin College, \\ Brunswick, ME 04011, U.S.A. \\ E-mail: naculich@bowdoin.edu
}

ABSTRACT: Inspired by recent developments on scattering equations, we present a constructive procedure for computing symmetric, amplitude-encoded, BCJ numerators for $n$ point gauge-theory amplitudes, thus satisfying the three virtues identified by Broedel and Carrasco. We also develop a constructive procedure for computing symmetric, amplitudeencoded dual-trace functions $\tau$ for $n$-point amplitudes. These can be used to obtain symmetric kinematic numerators that automatically satisfy color-kinematic duality. The $S_{n}$ symmetry of $n$-point gravity amplitudes formed from these symmetric dual-trace functions is completely manifest. Explicit expressions for four- and five-point amplitudes are presented.

KEYWORDS: Gauge-gravity correspondence, Scattering Amplitudes

ARXIV EPRINT: 1404.7141

\footnotetext{
${ }^{1}$ Research supported in part by the National Science Foundation under Grant No. PHY10-67961.
} 


\section{Contents}

1 Introduction 1

2 Review of color-kinematic duality $\quad 4$

$\begin{array}{lll}2.1 & \text { Double-color scalar amplitudes } & 6\end{array}$

$\begin{array}{ll}2.2 & \text { Gauge-theory amplitudes }\end{array}$

2.3 Gravity amplitudes 9

3 Virtuous kinematic numerators $\quad 9$

$\begin{array}{llr}3.1 & \text { Four-point symmetric kinematic numerators } & 10\end{array}$

$\begin{array}{lll}3.2 & \text { Five-point symmetric numerators } & 12\end{array}$

4 Virtuous dual-trace functions $\quad 13$

$\begin{array}{lll}4.1 & \text { Four-point symmetric dual-trace functions } & 16\end{array}$

$\begin{array}{lll}4.2 & \text { Five-point symmetric dual-trace functions } & 17\end{array}$

$\begin{array}{llr}5 & \text { Discussion } & 18\end{array}$

$\begin{array}{ll}\text { A Generating symmetric kinematic numerators } & 19\end{array}$

\section{Introduction}

Recent exciting work by Cachazo, He, and Yuan [1-4] has shed new light on the colorkinematic duality of gauge-theory amplitudes and the double-copy construction that relates gauge-theory and gravity amplitudes [5, 6]. Bern, Carrasco, and Johansson showed that tree-level gauge-theory and gravity amplitudes may be expressed as sums over cubic diagrams

$$
\mathcal{A}=\sum_{i} \frac{c_{i} n_{i}}{d_{i}}, \quad \mathcal{M}=\sum_{i} \frac{\tilde{n}_{i} n_{i}}{d_{i}}
$$

where the kinematic numerators $n_{i}$ are chosen to possess the same symmetries as the color factors $c_{i}$. Cachazo et al. [4] extended the gauge-gravity nexus to include amplitudes of the theory of massless scalars in the adjoint representation of $\mathrm{U}(N) \times \mathrm{U}(\tilde{N})$ with cubic interactions

$$
\mathcal{A}^{\text {scalar }}=\sum_{i} \frac{\tilde{c}_{i} c_{i}}{d_{i}}
$$

and they showed that tree-level $n$-point scattering amplitudes for all three theories may be expressed in terms of an integral over the space of $n$ marked points on a sphere, $\left\{\sigma_{j} \mid j=\right.$ 
$1, \cdots n\}$. For example, the double-color scalar theory amplitudes may be expressed as ${ }^{1}$

$$
\begin{aligned}
\mathcal{A}^{\text {scalar }} & =\sum_{\alpha, \beta} \operatorname{Tr}[\alpha] m(\alpha \mid \beta) \widetilde{\operatorname{Tr}}[\beta] \\
\operatorname{Tr}[\alpha] & =\operatorname{Tr}\left(T^{\mathrm{a}_{\alpha(1)}} T^{\mathrm{a}_{\alpha(2)}} \cdots T^{\mathrm{a}_{\alpha(n)}}\right) \\
m(\alpha \mid \beta) & =(-1)^{n-1} \int \frac{d^{n} \sigma}{\operatorname{vol} \mathrm{SL}(2, \mathbb{C})} \frac{\prod_{j}^{\prime} \delta\left(\sum_{k \neq j} \frac{s_{j k}}{\sigma_{j, k}}\right)}{\left(\sigma_{\alpha(1), \alpha(2)} \cdots \sigma_{\alpha(n), \alpha(1)}\right)\left(\sigma_{\beta(1), \beta(2)} \cdots \sigma_{\beta(n), \beta(1)}\right)}
\end{aligned}
$$

where $\sigma_{j, k}=\sigma_{j}-\sigma_{k}$ and $s_{j k}=\left(p_{j}+p_{k}\right)^{2}$ with $p_{j}$ the momenta of the external particles. The delta function localizes the integral (1.5) on the solutions of the scattering equations [1,2]

$$
\sum_{k \neq j} \frac{s_{j k}}{\sigma_{j, k}}=0 \quad \text { for } \quad j=1, \cdots n
$$

As these equations have, up to $\operatorname{SL}(2, \mathbb{C})$ transformations, $(n-3)$ ! solutions $\left\{\sigma_{j}^{(I)}\right\}$, the double-partial amplitude $m(\alpha \mid \beta)$ may expressed as a sum over solutions

$$
m(\alpha \mid \beta)=(-1)^{n-1} \sum_{I=1}^{(n-3) !} \frac{1}{\left(\sigma_{\alpha(1), \alpha(2)}^{(I)} \cdots \sigma_{\alpha(n), \alpha(1)}^{(I)}\right)\left(\sigma_{\beta(1), \beta(2)}^{(I)} \cdots \sigma_{\beta(n), \beta(1)}^{(I)}\right) \operatorname{det}^{\prime} \Phi\left(\sigma^{(I)}\right)}
$$

demonstrating that $m(\alpha \mid \beta)$ has rank $(n-3)$ !. The amplitudes (1.1) for gauge theory and gravity may also be succinctly expressed in terms of sums over solutions [3, 4]. Related work on the scattering equations includes refs. [7-25].

Because the color factors $c_{i}$ in eq. (1.1) satisfy Jacobi identities and hence are not linearly independent, the kinematic numerators $n_{i}$ for a given $n$-point gauge-theory amplitude are not uniquely determined. This generalized gauge freedom can be used to require that $n_{i}$ satisfy the same Jacobi identities as the color factors [5]. As a consequence all kinematic numerators $n_{i}$ may be expressed in terms of an independent set $\mathbf{n}_{1 \gamma n}$ associated with half-ladder diagrams. Defining color-ordered amplitudes $A_{\alpha}$ as the coefficients of the gauge-theory amplitude expressed in the trace basis

$$
\mathcal{A}=\sum_{\alpha} \operatorname{Tr}[\alpha] A_{\alpha}
$$

it can then be shown that the half-ladder numerators must satisfy

$$
A_{\alpha}=\sum_{\gamma} m(\alpha \mid 1 \gamma n) \mathbf{n}_{1 \gamma n}
$$

Because there are $(n-2)$ ! independent half-ladder numerators $\mathbf{n}_{1 \gamma n}$ and because rank $m=$ $(n-3)$ !, this equation cannot be uniquely inverted, meaning that $\mathbf{n}_{1 \gamma n}$ still possesses some residual generalized gauge freedom even after color-kinematic duality is imposed. Many different representations of the kinematic numerators are possible, e.g., see refs. [26-28].

\footnotetext{
${ }^{1}$ The definition of $m(\alpha \mid \beta)$ here differs from that of ref. [4] by an overall sign when $n$ is even.
} 
In an effort to define an economical and natural representation for the numerators, Broedel and Carrasco [29] enumerated three virtues that kinematic numerators would ideally possess: (1) color-kinematic duality (numerators obey the same symmetries as the associated color factors), (2) amplitude-encoding (external-state dependence is expressed in terms of color-ordered amplitudes $A_{\alpha}$ ), and (3) symmetry (numerator functions corresponding to diagrams with the same topology but different labelings of the external legs are all related by permutations of their arguments). They then proceeded to construct virtuous numerators for four- and five-point tree-level amplitudes, and for six-point MHV amplitudes in four dimensions, by assuming a general ansatz for the numerators and imposing functional constraints. Their approach becomes impracticable, however, for larger values of $n$, and they voiced the hope that a constructive procedure for virtuous numerators for arbitrary $n$-point amplitudes could be found.

In section 3 of this paper, we present a procedure to produce $n$-point kinematic numerators satisfying all three virtues of Broedel and Carrasco. We begin with a specific set of nonsymmetric numerators from ref. [4] obtained using the properties of $m(\alpha \mid \beta)$. By applying arbitrary permutations to the external legs, we derive other nonsymmetric sets of numerators. Finally, we generate a symmetric numerator by summing over all such representations. ${ }^{2}$ Our expression manifestly satisfies all Jacobi identities and diagram symmetries for the numerators without having to invoke the BCJ relations for the color-ordered amplitudes. We give explicit results for four- and five-point amplitudes, but the procedure is completely general.

The double-copy construction relates gauge-theory to gravity amplitudes by replacing the color factor $c_{i}$ in eq. (1.1) with a function $\tilde{n}_{i}$ of kinematic variables [5]. Bern and Dennen [31] suggested that, in a similar way, the gravity amplitude could be obtained from the gauge-theory amplitude (1.8) by replacing the color trace factor $\operatorname{Tr}[\alpha]$ with a dual-trace function $\tau_{\alpha}$ of the kinematic variables

$$
\mathcal{M}=\sum_{\alpha} \tau_{\alpha} A_{\alpha}
$$

It can then be shown that

$$
A_{\alpha}=\sum_{\beta} m(\alpha \mid \beta) \tau_{\beta} .
$$

Once again, because there are $(n-1) ! / 2$ independent $\tau_{\alpha}$ and rank $m=(n-3)$ !, this equation cannot be uniquely inverted; many choices of dual-trace functions are possible.

In ref. [31], Bern and Dennen presented explicit expressions for $\tau_{\alpha}$ in terms of $\mathbf{n}_{1 \gamma n}$ for four-, five-, and six-point amplitudes, and verified the existence of such expressions through nine points, but did not present a general procedure for arbitrary $n$. Further progress was achieved by Du et al. [32, 33].

In section 4 of this paper, we present a procedure to generate $n$-point dual-trace functions $\tau_{\alpha}$ that possess the three virtues identified by Broedel and Carrasco: (1) they automatically lead to kinematic numerators that obey color-kinematic duality, (2) they are

\footnotetext{
${ }^{2}$ Recently, Fu, Du, and Feng [30] presented a different but apparently equivalent algorithm by obtaining symmetric numerators from a KLT expression for the gauge-theory amplitude.
} 
expressed in terms of $A_{\alpha}$, i.e., they are amplitude-encoded, and (3) they are symmetric: a single function $\tau$ suffices to determine the full set via permutations of its arguments. We first identify a specific set of nonsymmetric dual-trace functions that satisfy eq. (1.11), apply arbitrary permutations to the external legs to obtain other representations, and then generate a symmetric dual-trace function by summing over all such representations. We present explicit expressions for four- and five-point functions, but again the procedure is completely general.

The expressions for $\tau_{\alpha}$ obtained in ref. [31] were observed to obey Kleiss-Kuijf relations, and the authors argued that this was a necessary condition for symmetric dual-trace functions expressed in terms of kinematic numerators. The results for $\tau_{\alpha}$ obtained in this paper do not satisfy Kleiss-Kuijf relations; they are expressed directly in terms of color-ordered amplitudes, and so are able to avoid this requirement. Thus, even after the virtues of Broedel and Carrasco are imposed, there remains some freedom in the dual-trace function.

This paper is structured as follows. Section 2 reviews the tree-level amplitudes of gauge, gravity, and double-color scalar theories, and the color-kinematic dualities that relate them. Section 3 describes the criteria of Broedel and Carrasco for virtuous numerators, outlines our procedure for constructing them, and presents explicit results for four- and five-point amplitudes. Section 4 reviews the properties of the dual-trace functions of Bern and Dennen, and then outlines our procedure for constructing them, again presenting explicit results for four- and five-point amplitudes. Section 5 discusses open questions, and an appendix contains the proof of the procedure used to produce symmetric kinematic numerators and dual-trace functions.

\section{Review of color-kinematic duality}

In this section, we review tree-level amplitudes of gauge, gravity, and double-color scalar theories, and the color-kinematic dualities that relate these various amplitudes.

We begin with the tree-level $n$-gluon amplitude $\mathcal{A}(1,2, \cdots, n)$, where the arguments denote the momentum $p_{j}$, polarization $\varepsilon_{j}$, and color $\mathrm{a}_{j}$ of the external particles $j=1, \cdots, n$. The color dependence can be specified in terms of a set of color factors $c_{i}$ obtained by sewing together cubic vertices $f_{\mathrm{abc}} \equiv \operatorname{Tr}\left(T^{\mathrm{a}}\left[T^{\mathrm{b}}, T^{\mathrm{c}}\right]\right)$, where $T^{\mathrm{a}}$ denote generators in the fundamental representation of the color group. The $n$-point amplitude is then expressed as a sum over all cubic diagrams [5]

$$
\mathcal{A}(1,2, \cdots, n)=\sum_{i} \frac{c_{i} n_{i}(1,2, \cdots, n)}{d_{i}(1,2, \cdots, n)} .
$$

The denominator $d_{i}(1,2, \cdots, n)$ associated with the color diagram $c_{i}$ is a product of the diagram propagators, and is a function of the external momenta $p_{j}$. The kinematic numerator $n_{i}(1,2, \cdots, n)$ associated with $c_{i}$ is a function of both $p_{j}$ and $\varepsilon_{j}$. (As usual, terms in the Feynman diagram expansion arising from quartic vertices can be parceled into terms involving only cubic vertices.) 
The color factors $c_{i}$ can be expanded in a trace basis

$$
c_{i}=\sum_{\alpha \in S_{n} / \mathbb{Z}} M_{i \alpha} \operatorname{Tr}[\alpha], \quad \operatorname{Tr}[\alpha] \equiv \operatorname{Tr}\left(T^{\mathrm{a}_{\alpha(1)}} T^{\mathrm{a}_{\alpha(2)}} \cdots T^{\mathrm{a}_{\alpha(n)}}\right) .
$$

The trace basis is independent provided we restrict the sum to permutations of $n$ indices modulo cyclic permutations. The gauge-theory amplitude (2.1) can be decomposed in this basis

$$
\begin{aligned}
\mathcal{A}(1,2, \cdots, n) & =\sum_{\alpha \in S_{n} / \mathbb{Z}} \operatorname{Tr}[\alpha] A_{\alpha}(1,2, \cdots, n), \\
A_{\alpha}(1,2, \cdots, n) & =\sum_{i} \frac{M_{i \alpha} n_{i}(1,2, \cdots, n)}{d_{i}(1,2, \cdots, n)}
\end{aligned}
$$

where the arguments of the color-ordered amplitudes $A_{\alpha}(1,2, \cdots, n)$ denote $p_{j}$ and $\varepsilon_{j}$. Since the trace basis is independent, the color-ordered amplitudes are well-defined and gauge invariant. Because of the invariance of the full amplitude $\mathcal{A}(1,2, \cdots, n)$ under permutations of the arguments and the independence of the trace basis, ${ }^{3}$ the color-ordered amplitudes are all related to one another via

$$
A_{\alpha}(1,2, \cdots, n)=A(\alpha(1), \cdots, \alpha(n))
$$

where $A(i, j, \cdots) \equiv A_{12 \cdots n}(i, j, \cdots)$, the coefficient of $\operatorname{Tr}\left(T^{\mathrm{a}_{1}} T^{\mathrm{a}_{2}} \cdots T^{\mathrm{a}_{n}}\right)$. The color-ordered amplitudes are thus "symmetric" functions, in the sense of Broedel and Carrasco [29].

The color factors $c_{i}$ in eq. (2.1) are not independent but satisfy various Jacobi identities

$$
c_{i}+c_{j}+c_{k}=0
$$

which can be expressed [34-36] as $\sum_{i} \ell_{i} c_{i}=0$, where $\ell_{i}$ are left null vectors of the matrix $M_{i \alpha}: \sum_{i} \ell_{i} M_{i \alpha}=0$. The matrix $M_{i \alpha}$ has rank $(n-2)$ ! so there are $(n-2)$ ! independent color factors. The matrix $M_{i \alpha}$ also possesses a set of right null vectors $\sum_{\alpha} M_{i \alpha} r_{\alpha}=0$. By eq. (2.4), these give rise [34-36] to a set of constraints $\sum_{\alpha} A_{\alpha} r_{\alpha}=0$ on the color-ordered amplitudes, viz., the Kleiss-Kuijf relations [37].

Because of the linear dependence of the color factors $c_{i}$, the kinematic numerators $n_{i}(1,2, \cdots, n)$ in eq. (2.1) are not uniquely determined but can undergo what are termed generalized gauge transformations [38] without altering the amplitudes. The insight of Bern, Carrasco, and Johannson [5] is that there exists a generalized gauge choice for which the numerators satisfy the same Jacobi identities as the color factors

$$
n_{i}(1,2, \cdots, n)+n_{j}(1,2, \cdots, n)+n_{k}(1,2, \cdots, n)=0 .
$$

We will refer to a set of kinematic numerators satisfying eq. (2.7) as BCJ numerators. Such a choice is not unique: there remain residual generalized gauge transformations that preserve the Jacobi identities (2.7).

\footnotetext{
${ }^{3}$ See discussion in the appendix.
} 
A subset of the color factors $c_{i}$ are the half-ladder diagrams, labeled by $\alpha \in S_{n}$ :

$$
\begin{aligned}
\mathbf{c}_{\alpha} & =\sum_{\mathrm{b}_{1}, \ldots, \mathrm{b}_{n-3}} f_{\mathrm{a}_{\alpha(1)} \mathrm{a}_{\alpha(2)} \mathrm{b}_{1} \cdots f_{\mathrm{b}_{n-3} \mathrm{a}_{\alpha(n-1)} \mathrm{a}_{\alpha(n)}}} \\
& =\operatorname{Tr}\left(T^{\mathrm{a}_{\alpha(1)}}\left[T^{\mathrm{a}_{\alpha(2)}},\left[\cdots\left[T^{\mathrm{a}_{\alpha(n-1)}}, T^{\mathrm{a}_{\alpha(n)}}\right] \cdots\right]\right]\right) .
\end{aligned}
$$

An independent set of color factors $[39,40]$ consists of those half ladders with $\alpha(1)=1$ and $\alpha(n)=n$ :

$$
\mathbf{c}_{1 \gamma n} \equiv \mathbf{c}_{1 \gamma(2) \cdots \gamma(n-1) n}, \quad \gamma \in S_{n-2}
$$

whose expansion in the trace basis begins

$$
\mathbf{c}_{1 \gamma n}=\operatorname{Tr}[1 \gamma(2) \cdots \gamma(n-1) n]+(-1)^{n} \operatorname{Tr}[n \gamma(n-1) \cdots \gamma(1) 1]+\cdots
$$

where $+\cdots$ denotes traces without 1 and $n$ adjacent. That the set $\mathbf{c}_{1 \gamma n}$ is independent follows from eq. (2.10) together with the independence of the trace basis (modulo cyclic permutations). That the set is complete was shown in ref. [40] where an arbitrary color diagram was reduced to a linear combination $c_{i}=\sum_{i} \lambda_{i, \gamma} \mathbf{c}_{1 \gamma n}$ using the Jacobi identities. By matching the coefficients of $\operatorname{Tr}[1 \gamma(2) \cdots \gamma(n-1) n]$ on both sides of this equation, one then establishes that

$$
c_{i}=\sum_{\gamma \in S_{n-2}} M_{i, 1 \gamma n} \mathbf{c}_{1 \gamma n} .
$$

Then eqs. (2.1) and (2.4) imply [39, 40]

$$
\mathcal{A}(1,2, \cdots, n)=\sum_{\gamma \in S_{n-2}} \mathbf{c}_{1 \gamma n} A(1, \gamma(2), \cdots, \gamma(n-1), n) .
$$

\subsection{Double-color scalar amplitudes}

In their seminal paper [5], Bern, Carrasco, and Johansson showed that, given a set of BCJ numerators $n_{i}(1,2, \cdots, n)$, one can obtain tree-level gravity amplitudes ${ }^{4}$ by replacing $c_{i}$ in eq. (2.1) with the kinematic numerators $\tilde{n}_{i}(1,2, \cdots, n)$ of a second gauge theory (the double-copy procedure)

$$
\mathcal{M}(1,2, \cdots, n)=\sum_{i} \frac{\tilde{n}_{i}(1,2, \cdots, n) n_{i}(1,2, \cdots, n)}{d_{i}(1,2, \cdots, n)}
$$

where the $\tilde{n}_{i}(1,2, \cdots, n)$ need not themselves satisfy the Jacobi identities [38].

An alternative double-copy procedure replaces the $n_{i}(1,2, \cdots, n)$ in eq. $(2.1)$ with the color factors $\tilde{c}_{i}$ of a second color group

$$
\mathcal{A}^{\text {scalar }}(1,2, \cdots, n)=\sum_{i} \frac{\tilde{c}_{i} c_{i}}{d_{i}(1,2, \cdots, n)} .
$$

Such an expression corresponds [4] to the $n$-point amplitude of a theory of massless scalar particles $\phi^{\text {aa' }}$ in the adjoint of the color group $\mathrm{U}(N) \times \mathrm{U}(\tilde{N})$ with cubic interactions of the form

$$
f_{\mathrm{abc}} \tilde{f}_{\mathrm{a}^{\prime} \mathrm{b}^{\prime} \mathrm{c}^{\prime}} \phi^{\mathrm{aa} \mathrm{a}^{\prime}} \phi^{\mathrm{bb}{ }^{\prime}} \phi^{\mathrm{cc}{ }^{\prime}}
$$

\footnotetext{
${ }^{4} \mathrm{Up}$ to an overall factor depending on coupling strengths that we suppress throughout this paper.
} 
where $f_{\mathrm{abc}}$ and $\tilde{f}_{\mathrm{a}^{\prime} \mathrm{b}^{\prime} \mathrm{c}^{\prime}}$ are the structure constants of $\mathrm{U}(N)$ and $\mathrm{U}(\tilde{N})$. Using

$$
\tilde{c}_{i}=\sum_{\alpha \in S_{n} / \mathbb{Z}} M_{i \alpha} \widetilde{\operatorname{Tr}}[\alpha], \quad \widetilde{\operatorname{Tr}}[\alpha] \equiv \operatorname{Tr}\left(\tilde{T}^{\mathrm{a}_{\alpha(1)}} \tilde{T}^{\mathrm{a}_{\alpha(2)}} \cdots \tilde{T}^{\mathrm{a}_{\alpha(n)}}\right)
$$

together with eq. (2.2), the double-color amplitude (2.14) can be written as

$$
\mathcal{A}^{\text {scalar }}(1,2, \cdots, n)=\sum_{\alpha \in S_{n} / \mathbb{Z}} \sum_{\beta \in S_{n} / \mathbb{Z}} \operatorname{Tr}[\alpha] m(\alpha \mid \beta) \widetilde{\operatorname{Tr}}[\beta]
$$

where $^{5}$

$$
m(\alpha \mid \beta)=\sum_{i} \frac{M_{i \alpha} M_{i \beta}}{d_{i}(1,2, \cdots, n)}, \quad \alpha, \beta \in S_{n} .
$$

The coefficients $A_{\alpha}$ of $\operatorname{Tr}[\alpha]$ of the gauge-theory amplitude (2.3) are sometimes termed "partial amplitudes." In ref. [4], Cachazo, He, and Yuan dubbed $m(\alpha \mid \beta)$, the coefficients of $\operatorname{Tr}[\alpha] \widetilde{\operatorname{Tr}}[\beta]$ in eq. (2.17), "double-partial amplitudes." They showed that $m(\alpha \mid \beta)$ computes the sum of all trivalent scalar diagrams that can be regarded both as $\alpha$-color-ordered and $\beta$-color-ordered, where each diagram's contribution is given by the product of its propagators. The double-partial amplitudes satisfy (on both sides) Kleiss-Kuijf relations $\sum_{\alpha} r_{\alpha} m(\alpha \mid \beta)=\sum_{\alpha} m(\alpha \mid \beta) r_{\beta}=0$ where $r_{\alpha}$ are right null vectors of the rank $(n-2)$ ! matrix $M_{i \alpha}$.

Using eq. (2.11), the double-color amplitude (2.14) can also be expressed as

$$
\mathcal{A}^{\text {scalar }}(1,2, \cdots, n)=\sum_{\gamma \in S_{n-2}} \sum_{\delta \in S_{n-2}} \tilde{\mathbf{c}}_{1 \gamma n} m(1 \gamma n \mid 1 \delta n) \mathbf{c}_{1 \delta n} .
$$

The double-partial amplitudes in this equation, $m(1 \gamma n \mid 1 \delta n)$, are essentially the entries in the $(n-2) ! \times(n-2) !$ matrix considered in ref. [41]. There it was shown by consideration of low values of $n$ that, as a result of momentum conservation, the rank of this matrix is $(n-3)$ !. Cachazo et al. showed that the double-partial amplitudes could alternatively be expressed as eq. (1.7), which shows explicitly that $m(\alpha \mid \beta)$ has rank $(n-3)$ !. It consequently possesses $(n-3) !-(n-2)$ ! additional null vectors, dependent on kinematic invariants, which implies that the double-partial amplitudes satisfy BCJ relations [5] in addition to Kleiss-Kuijf relations.

\subsection{Gauge-theory amplitudes}

Just as the Jacobi identities for the color factors (2.6) imply that they can be expressed as a linear combination of independent half-ladder color factors (2.11), so the Jacobi identities (2.7) imply that the kinematic numerators can be written in terms of an independent basis of numerators $\mathbf{n}_{1 \gamma n}(1,2, \cdots, n)$ associated with $\mathbf{c}_{1 \gamma n}$

$$
n_{i}(1,2, \cdots, n)=\sum_{\gamma \in S_{n-2}} M_{i, 1 \gamma n} \mathbf{n}_{1 \gamma n}(1,2, \cdots, n)
$$

\footnotetext{
${ }^{5}$ This definition of $m(\alpha \mid \beta)$ differs by a sign from that of ref. [4] when $n$ is even.
} 
Then, analogous to eq. (2.19) for double-color scalar amplitude, we can write the gaugetheory amplitude (2.1) as

$$
\mathcal{A}(1,2, \cdots, n)=\sum_{\gamma \in S_{n-2}} \sum_{\delta \in S_{n-2}} \mathbf{c}_{1 \gamma n} m(1 \gamma n \mid 1 \delta n) \mathbf{n}_{1 \delta n} .
$$

We can also substitute eq. (2.20) into eq. (2.4) to obtain the color-ordered amplitudes

$$
A_{\alpha}(1,2, \cdots, n)=\sum_{\gamma \in S_{n-2}} m(\alpha \mid 1 \gamma n) \mathbf{n}_{1 \gamma n}(1,2, \cdots, n) .
$$

As noted above, the matrix $m(\alpha \mid 1 \gamma n)$ has rank $(n-3)$ ! and has $(n-2) !-(n-3)$ ! additional $^{6}$ null vectors (dependent on kinematic invariants) which give rise $[7,41,42]$ to the BCJ relations [5] among the color-ordered amplitudes.

The kernel of the matrix corresponds to $(n-2) !-(n-3)$ ! degrees of freedom of residual generalized gauge transformations of the BCJ numerators (i.e. generalized gauge transformations that preserve the Jacobi constraints (2.7)). Because of this residual gauge freedom, eq. (2.22) cannot be inverted to obtain unique expressions for BCJ numerators in terms of color-ordered amplitudes. To invert eq. (2.22), we must first make a choice of gauge. One possible gauge choice is to set

$$
\mathbf{n}_{1 \gamma(2) \cdots \gamma(n-1) n}=0, \quad \gamma(n-1) \neq n-1
$$

which allows us to restrict the sum in eq. (2.22) to $S_{n-3}$.

Now consider the subset of color-ordered amplitudes

$$
\begin{aligned}
A(1, \beta(2), \cdots, & \beta(n-2), n, n-1) \\
& =\sum_{\gamma \in S_{n-3}} m(1 \beta n, n-1 \mid 1 \gamma n-1, n) \mathbf{n}_{1 \gamma(2) \cdots \gamma(n-2) n-1, n}(1,2, \cdots, n) .
\end{aligned}
$$

It was shown in ref. [4] by using KLT orthogonality [2] that the $(n-3) ! \times(n-3)$ ! submatrix appearing in eq. (2.24) is invertible, with the inverse given by the (negative of the) momentum kernel ${ }^{7}[27,43-45]$

$$
S[\gamma \mid \beta]=\prod_{i=2}^{n-2}\left[s_{1, \gamma(i)}+\sum_{j=2}^{i-1} \theta(\gamma(j), \gamma(i))_{\beta} s_{\gamma(j), \gamma(i)}\right] .
$$

Thus the nonzero members of the independent basis of numerators can be expressed as [4]

$$
\mathbf{n}_{1 \gamma(2) \cdots \gamma(n-2) n-1, n}(1,2, \cdots, n)=-\sum_{\beta \in S_{n-3}} S[\gamma \mid \beta] A(1, \beta(2), \cdots, \beta(n-2), n, n-1) .
$$

The full set of (BCJ) numerators, including the half-ladders not included in the independent set, can then be obtained from eqs. (2.23) and (2.26) via eq. (2.20).

\footnotetext{
${ }^{6}$ In addition, that is, to the null vectors of $M_{i \alpha}$ which give rise to the Kleiss-Kuijf relations.

${ }^{7}$ Here $\gamma, \beta \in S_{n-3}$ are permutations acting on labels $2,3, \ldots, n-2 ; \theta(r, s)_{\beta}=1$ if the ordering of $r, s$ is the same in both sequences of labels, $\gamma(2), \ldots, \gamma(n-2)$ and $\beta(2), \ldots, \beta(n-2)$, and zero otherwise.
} 


\subsection{Gravity amplitudes}

By the double-copy construction, we can replace $\mathbf{c}_{1 \gamma n}$ in eqs. (2.12) and (2.21) with $\mathbf{n}_{1 \gamma n}(1,2, \cdots, n)$ to obtain the gravity amplitude

$$
\begin{aligned}
\mathcal{M}(1,2, \cdots, n) & =\sum_{\gamma \in S_{n-2}} \mathbf{n}_{1 \gamma n}(1,2, \cdots, n) A(1, \gamma(2), \cdots, \gamma(n-1), n) \\
& =\sum_{\gamma \in S_{n-2}} \sum_{\delta \in S_{n-2}} \mathbf{n}_{1 \gamma n}(1,2, \cdots, n) m(1 \gamma n \mid 1 \delta n) \mathbf{n}_{1 \delta n}(1,2, \cdots, n) .
\end{aligned}
$$

The specific choice of gauge in eqs. (2.23) and (2.26) can then be used to write

$$
\begin{aligned}
\mathcal{M}(1,2, \cdots, n)= & \sum_{\gamma \in S_{n-3}} \sum_{\delta \in S_{n-3}} A(1, \gamma(2), \cdots, \gamma(n-2), n-1, n) S[\gamma \mid \delta] \\
& \times A(1, \delta(2), \cdots, \delta(n-2), n, n-1)
\end{aligned}
$$

which is one possible form of the field-theory limit of the KLT relation [27, 46, 47].

\section{$3 \quad$ Virtuous kinematic numerators}

As described in the previous section, even after requiring the kinematic numerators to satisfy color-kinematic duality, a certain amount of generalized gauge freedom remains. To fix this residual gauge freedom in a natural, economical, and possibly unique way, Broedel and Carrasco [29] identified three desirable features that a set of numerators should possess:

1. color-kinematic duality: BCJ numerators obey the same symmetries as their associated color factors. Such numerators can be used to construct gravity amplitudes using the double-copy procedure.

2. amplitude-encoding: the external state dependence (e.g., helicities) of the numerators is expressed in terms of color-ordered amplitudes. Such a representation would also be independent of the number of space-time dimensions.

3. symmetry: all of the numerators $n_{i}$ for a given diagram topology can be expressed via permutations of the arguments of a single function. This virtue is thus one of economy, and also makes the gravity amplitudes constructed from these numerators manifestly invariant under permutations of the external legs.

Kinematic numerators that satisfy all three features are dubbed "virtuous."

As we saw in the previous section, numerators that satisfy the first virtue are expressed in terms of an independent basis of half-ladder numerators $\mathbf{n}_{1 \gamma n}(1,2, \cdots, n)$ via eq. (2.20). Color-ordered amplitudes are expressed as in terms of these as

$$
A_{\alpha}(1,2, \cdots, n)=\sum_{\gamma \in S_{n-2}} m(\alpha \mid 1 \gamma n) \mathbf{n}_{1 \gamma n}(1,2, \cdots, n) .
$$

Were we able to invert this equation, we would possess numerators that also satisfy the second virtue of amplitude-encoding. Because $\operatorname{det} m=0$, eq. (3.1) has no unique inverse; there 
exists rather a family of generalized inverses [42, 48], each corresponding to a particular gauge choice for $\mathbf{n}_{1 \gamma n}$. One such choice is given by [4]

$$
\begin{aligned}
\mathbf{n}_{1 \gamma(2) \cdots \gamma(n-1) n}(1,2, \cdots, n) & =0, \\
\mathbf{n}_{1 \gamma(2) \cdots \gamma(n-2) n-1, n}(1,2, \cdots, n) & =-\sum_{\beta \in S_{n-3}} S[\gamma \mid \beta] A(1, \beta(2), \cdots, \beta(n-2), n, n-1) .
\end{aligned}
$$

This choice, however, does not satisfy the third virtue of symmetry.

Next, we describe how one can generate a symmetric representation of numerators starting from a nonsymmetric representation, such as that given in eq. (3.2). ${ }^{8}$ We show in the appendix that, given one set of numerators $\mathbf{n}_{\alpha}(1,2, \cdots, n)$, one can use an arbitrary permutation $\beta$ acting on the external legs to generate another set of valid numerators

$$
\mathbf{n}_{\alpha}^{\prime}(1,2, \cdots, n)=\mathbf{n}_{\beta^{-1} \alpha}(\beta(1), \cdots, \beta(n))
$$

i.e., these also satisfy eq. (3.1). If we average over all permutations $\beta \in S_{n}$, the resulting function

$$
\mathbf{n}(1,2, \cdots, n) \equiv \mathbf{n}^{\mathrm{sym}}(1,2, \cdots, n)=\frac{1}{n !} \sum_{\beta \in S_{n}} \mathbf{n}_{\beta^{-1}}(\beta(1), \cdots, \beta(n))
$$

will be symmetric; i.e., all half-ladder numerators are given by permutations of the arguments of the single function $\mathbf{n}(1,2, \cdots, n)$. Symmetric numerator functions for topologies other than the half-ladder are then obtained from $\mathbf{n}(1,2, \cdots, n)$ via eq. (2.20). Since the individual sets of numerators $\mathbf{n}_{\alpha}^{\prime}(1,2, \cdots, n)$ satisfy eq. (3.1), so does the average (3.4):

$$
A(\alpha(1), \cdots, \alpha(n))=\sum_{\gamma \in S_{n-2}} m(\alpha \mid 1 \gamma n) \mathbf{n}(1, \gamma(2), \cdots, \gamma(n-1), n) .
$$

Hence we have constructed a representation of kinematic numerators satisfying all three virtues of Broedel and Carrasco.

We apply this procedure in the following subsections to obtain explicit expressions for virtuous numerators for four- and five-point amplitudes. The expressions become increasingly lengthy for higher-point amplitudes, but the important point is that there exists a constructive proof of the existence of virtuous numerators for all tree-level $n$-gluon amplitudes. Whether such numerators are unique remains an open question.

In a recent paper [30], Fu, Du, and Feng also gave a prescription for obtaining virtuous numerators based on a similar symmetrization strategy applied to a KLT expression for the gauge-theory amplitude. It seems likely that this gives the same results as eq. (3.4). We will compare specific results below.

\subsection{Four-point symmetric kinematic numerators}

For the four-point amplitude, there is only one topology for color factors, the half ladder. A symmetric half-ladder numerator $\mathbf{n}(1,2,3,4)$ must satisfy eq. (3.5) which in this

\footnotetext{
${ }^{8}$ We thank Freddy Cachazo for suggesting this approach.
} 
case becomes

$$
A(1,2,3,4)=\left(\frac{1}{s_{12}}+\frac{1}{s_{14}}\right) \mathbf{n}(1,2,3,4)+\frac{1}{s_{14}} \mathbf{n}(1,3,4,2) .
$$

Equation (3.2) yields a nonsymmetric amplitude-encoded BCJ representation for independent half-ladder numerators

$$
\begin{aligned}
& \mathbf{n}_{1234}(i, j, k, l)=-s_{i j} A(i, j, l, k), \\
& \mathbf{n}_{1324}(i, j, k, l)=0
\end{aligned}
$$

with the remaining numerators given by eq. (2.20), i.e., by Jacobi identities and numerator symmetries. We obtain a virtuous representation by summing over all permutations (3.4)

$$
\begin{aligned}
\mathbf{n}(1,2,3,4)=\frac{1}{12}\left(s_{12}+\right. & \left.s_{34}\right)[A(1,2,3,4)-A(1,3,4,2)] \\
& +\frac{1}{12}\left(s_{13}+s_{24}-s_{14}-s_{23}\right) A(1,4,2,3) .
\end{aligned}
$$

We have reduced the number of terms by using the cyclic invariance of $A(1,2,3,4)$ as well as the reversal symmetry $A(1,2,3,4)=A(4,3,2,1)$. This expression manifestly obeys the Jacobi identities

$$
\mathbf{n}(1,2,3,4)+\mathbf{n}(1,3,4,2)+\mathbf{n}(1,4,2,3)=0
$$

as well as the dihedral symmetries of the half-ladder diagram

$$
\mathbf{n}(1,2,3,4)=-\mathbf{n}(2,1,3,4)=-\mathbf{n}(1,2,4,3)=\mathbf{n}(4,3,2,1) .
$$

Equation (3.8) also satisfies eq. (3.6) provided that the color-ordered amplitudes satisfy the four-point BCJ relations

$$
s_{i j} A(i, j, k, l)=s_{i k} A(i, l, j, k) .
$$

Our expression (3.8) agrees with that recently obtained in ref. [30]. It is also in agreement with the shorter expression in ref. [29]

$$
\mathbf{n}(1,2,3,4)=\frac{1}{3}\left[s_{12} A(1,2,3,4)-s_{14} A(1,4,2,3)\right]
$$

once momentum conservation and the Kleiss-Kuijf (subcyclic) identity $A(1,2,3,4)+$ $A(1,3,4,2)+A(1,4,2,3)=0$ are imposed. In fact, the three virtues listed above are sufficient to uniquely determine the four-point numerator.

The BCJ relations (3.11) can be used to rewrite eq. (3.12) as

$$
\mathbf{n}(1,2,3,4)=\frac{1}{3} s_{12}[A(1,2,3,4)-A(1,3,4,2)] .
$$

We could further use the BCJ relations to write $\mathbf{n}(1,2,3,4)$ in terms of a single color-ordered amplitude, but only at the price of having kinematic invariants in the denominator. 


\subsection{Five-point symmetric numerators}

For the five-point amplitude, there is again only one topology for the color factors, the half ladder. A symmetric half-ladder numerator $\mathbf{n}(1,2,3,4,5)$ satisfies eq. (3.5), which after using Jacobi identities is equivalent to

$$
\begin{aligned}
A(1,2,3,4,5)=\frac{\mathbf{n}(1,2,3,4,5)}{s_{12} s_{45}}+\frac{\mathbf{n}(2,3,4,5,1)}{s_{23} s_{51}} & \\
& +\frac{\mathbf{n}(3,4,5,1,2)}{s_{34} s_{12}}+\frac{\mathbf{n}(4,5,1,2,3)}{s_{45} s_{23}}+\frac{\mathbf{n}(5,1,2,3,4)}{s_{51} s_{34}} .
\end{aligned}
$$

Equation (3.2) yields a nonsymmetric amplitude-encoded BCJ representation for independent half-ladder numerators

$$
\begin{aligned}
& \mathbf{n}_{12345}(i, j, k, l, m)=-s_{i j}\left(s_{i k}+s_{j k}\right) A(i, j, k, m, l)-s_{i j} s_{i k} A(i, k, j, m, l), \\
& \mathbf{n}_{13245}(i, j, k, l, m)=-s_{i j} s_{i k} A(i, j, k, m, l)-s_{i k}\left(s_{i j}+s_{j k}\right) A(i, k, j, m, l), \\
& \mathbf{n}_{14235}(i, j, k, l, m)=0, \\
& \mathbf{n}_{12435}(i, j, k, l, m)=0, \\
& \mathbf{n}_{14235}(i, j, k, l, m)=0, \\
& \mathbf{n}_{14325}(i, j, k, l, m)=0
\end{aligned}
$$

where the other half-ladder numerators are obtained using eq. (2.20). Again, we obtain a virtuous representation by summing over all permutations (3.4)

$$
\begin{aligned}
\mathbf{n}(1,2, & 3,4,5) \\
= & \frac{1}{60}\left[\left(s_{12} s_{13}+2 s_{12} s_{23}+2 s_{34} s_{45}+s_{35} s_{45}\right) A(1,2,3,4,5)\right. \\
& +\left(s_{13} s_{14}+s_{12} s_{15}+s_{23} s_{24}+2 s_{23} s_{34}+s_{24} s_{34}+s_{25} s_{35}+s_{15} s_{45}\right) A(1,4,3,2,5) \\
& +\left(s_{13} s_{14}+s_{23} s_{24}+s_{12} s_{25}+2 s_{13} s_{34}+s_{14} s_{34}+s_{15} s_{35}+s_{25} s_{45}\right) A(1,3,4,2,5) \\
& +\left(s_{12} s_{14}-2 s_{12} s_{15}+2 s_{12} s_{24}-s_{12} s_{25}-s_{34} s_{45}+s_{35} s_{45}\right) A(1,2,4,3,5) \\
& +\left(s_{12} s_{14}+s_{13} s_{15}+s_{23} s_{25}+s_{24} s_{34}+2 s_{23} s_{35}+s_{25} s_{35}+s_{14} s_{45}\right) A(1,4,2,3,5) \\
& +\left(s_{12} s_{13}-s_{12} s_{23}-s_{14} s_{45}-2 s_{15} s_{45}+2 s_{24} s_{45}+s_{25} s_{45}\right) A(1,3,2,4,5) \\
& +\left(2 s_{12} s_{13}+s_{12} s_{23}+2 s_{34} s_{45}+s_{35} s_{45}\right) A(1,2,5,4,3) \\
& +\left(s_{12} s_{13}-s_{12} s_{23}-2 s_{14} s_{45}-s_{15} s_{45}+s_{24} s_{45}+2 s_{25} s_{45}\right) A(1,4,5,2,3) \\
& +\left(-s_{13} s_{15}-s_{12} s_{24}-s_{23} s_{25}-s_{14} s_{34}-2 s_{13} s_{35}-s_{15} s_{35}-s_{24} s_{45}\right) A(1,3,5,2,4) \\
& +\left(2 s_{12} s_{14}-s_{12} s_{15}+s_{12} s_{24}-2 s_{12} s_{25}-s_{34} s_{45}+s_{35} s_{45}\right) A(1,2,5,3,4) \\
& +\left(s_{12} s_{13}+2 s_{12} s_{23}+s_{34} s_{45}+2 s_{35} s_{45}\right) A(1,4,5,3,2) \\
& \left.+\left(2 s_{12} s_{13}+s_{12} s_{23}+s_{34} s_{45}+2 s_{35} s_{45}\right) A(1,3,5,4,2)\right]
\end{aligned}
$$


where we have used the reversal property $A(1,2,3,4,5)=-A(5,4,3,2,1)$ together with cyclic invariance of $A(1,2,3,4,5)$. This expression automatically obeys the dihedral symmetry of the half-ladder diagram

$$
\mathbf{n}(1,2,3,4,5)=-\mathbf{n}(2,1,3,4,5)=-\mathbf{n}(1,2,3,5,4)=-\mathbf{n}(5,4,3,2,1)
$$

as well as all the Jacobi identities.

Equation (3.16) agrees with the shorter expression given in Broedel and Carrasco [29] after imposing momentum conservation and BCJ relations on the color-ordered amplitudes. The Broedel-Carrasco expression is virtuous, but to show that it obeys eq. (3.17) requires the imposition of momentum conservation and BCJ relations, whereas for eq. (3.16) the dihedral symmetry is manifest. Both the Broedel-Carrasco expression and eq. (3.16) satisfy eq. (3.14) only after momentum conservation and the BCJ relations are imposed.

We can re-express eq. (3.16) in the Kleiss-Kuijf basis to find

$$
\begin{aligned}
& \mathbf{n}(1,2,3,4,5) \\
& =\frac{1}{10}\left[s_{12}\left(s_{13}+s_{23}\right)+s_{45}\left(s_{34}+s_{35}\right)\right] A(1,2,3,4,5) \\
& \quad+\frac{1}{60}\left[s_{12}\left(s_{13}+2 s_{14}-s_{23}-2 s_{25}\right)+s_{13}\left(s_{14}-s_{15}-2 s_{35}\right)+s_{23}\left(s_{24}-s_{25}+2 s_{34}\right)\right. \\
& \left.\quad+s_{34}\left(s_{24}-s_{14}\right)+s_{35}\left(s_{25}-s_{15}\right)+s_{45}\left(-2 s_{14}+2 s_{25}-s_{34}+s_{35}\right)\right] A(1,4,3,2,5) \\
& \quad+\frac{1}{60}\left[s_{12}\left(3 s_{13}-s_{24}+s_{25}\right)+s_{13}\left(s_{14}-s_{15}+2 s_{34}-2 s_{35}\right)+s_{23}\left(s_{24}-s_{25}\right)\right. \\
& \left.\quad+s_{45}\left(-2 s_{14}-s_{15}+3 s_{25}+2 s_{34}+s_{35}\right)\right] A(1,3,4,2,5) \\
& +\frac{1}{20}\left[s_{12}\left(s_{13}+s_{14}-s_{15}+s_{23}+s_{24}-s_{25}\right)+2 s_{35} s_{45}\right] A(1,2,4,3,5) \\
& +\frac{1}{60}\left[s_{12}\left(s_{13}+3 s_{14}-s_{15}+2 s_{23}-2 s_{25}\right)+s_{14}\left(s_{45}-s_{34}\right)\right. \\
& \left.\quad+s_{24}\left(s_{34}-s_{45}\right)+s_{35}\left(-2 s_{13}-s_{15}+2 s_{23}+s_{25}+3 s_{45}\right)\right] A(1,4,2,3,5) \\
& +\frac{1}{20}\left[2 s_{12} s_{13}+s_{45}\left(-s_{14}-s_{15}+s_{24}+s_{25}+s_{34}+s_{35}\right)\right] A(1,3,2,4,5) .
\end{aligned}
$$

This expression differs from the result given in ref. [30], but that result apparently contains some typographical errors which will be corrected in a revised version. ${ }^{9}$ We emphasize that eq. (3.18) manifestly obeys Jacobi identities and dihedral symmetries using only the Kleiss-Kuijf relations and the cyclic and reversal properties of the color-ordered amplitudes (but not momentum conservation or BCJ relations).

Fu et al. [30] also give explicit, rather lengthy, expressions for virtuous numerators for six-point amplitudes.

\section{Virtuous dual-trace functions}

The color factors $c_{i}$ and the kinematic numerators $n_{i}(1,2, \cdots, n)$ play dual roles in the gauge-theory amplitude (2.1). Bern and Dennen [31] proposed that a role dual to the

\footnotetext{
${ }^{9}$ Private communication.
} 
traces of generators $\operatorname{Tr}[\alpha]$ in eq. (2.3) could be played by a function $\tau_{\alpha}(1,2, \cdots, n)$ of the variables $p_{j}$ and $\varepsilon_{j}$ that is related to $n_{i}(1,2, \cdots, n)$ in the same way $(2.2)$ that $\operatorname{Tr}[\alpha]$ is related to $c_{i}$ :

$$
n_{i}(1,2, \cdots, n)=\sum_{\alpha \in S_{n} / \mathbb{Z}} M_{i \alpha} \tau_{\alpha}(1,2, \cdots, n) .
$$

Just as the Jacobi identities for the color factors (2.6) can be expressed as $\sum_{i} \ell_{i} c_{i}=0$, where $\ell_{i}$ are left null vectors of the rank $(n-2)$ ! matrix $M_{i \alpha}$, so the Jacobi identities for the kinematic numerators $(2.7)$ are expressed as $\sum_{i} \ell_{i} n_{i}=0$, and will therefore be automatically satisfied by eq. (4.1). Using eq. (4.1), together with eqs. (2.4) and (2.18), the color-ordered amplitudes can be written in terms of the dual-trace functions as

$$
A_{\alpha}(1,2, \cdots, n)=\sum_{\beta \in S_{n} / \mathbb{Z}} m(\alpha \mid \beta) \tau_{\beta}(1,2, \cdots, n) .
$$

If we could invert this equation, we would have a prescription for an amplitude-encoded dual-trace function, i.e. in which the dependence on the external states is expressed through the color-ordered amplitudes. Since $m(\alpha \mid \beta)$ has rank $(n-3)$ !, there is no unique inverse, but rather a family of generalized inverses, each corresponding to a particular gauge choice imposed on the dual-trace functions.

First we consider the symmetries of the dual-trace functions $\tau_{\alpha}$. Because $M_{i \alpha}=M_{i \alpha^{\prime}}$ when $\alpha$ and $\alpha^{\prime}$ are related by cyclic permutations, and $M_{i \alpha}=(-1)^{n} M_{i \alpha^{\prime}}$ when $\alpha^{\prime}$ is the reverse of $\alpha^{\prime}$, we can impose these properties on $\tau_{\alpha}$ without loss of generality

$$
\begin{aligned}
& \tau_{\alpha(1) \alpha(2) \cdots \alpha(n)}(1,2, \cdots, n)=\tau_{\alpha(2) \alpha(3) \cdots \alpha(1)}(1,2, \cdots, n) \\
& \tau_{\alpha(1) \alpha(2) \cdots \alpha(n)}(1,2, \cdots, n)=(-1)^{n} \tau_{\alpha(n) \cdots \alpha(2) \alpha(1)}(1,2, \cdots, n)
\end{aligned}
$$

leaving $(n-1) ! / 2$ independent dual-trace functions to be determined. These must satisfy eq. (4.1), which for the independent half-ladder numerators takes the form

$$
\begin{aligned}
& \mathbf{n}_{1 \gamma(2) \cdots \gamma(n-1) n}(1,2, \cdots, n) \\
& \quad=\tau_{1[\gamma(2),[\cdots,[\gamma(n-1), n] \cdots]]}(1,2, \cdots, n) \\
& \quad=\tau_{1 \gamma(2) \cdots \gamma(n-1) n}(1,2, \cdots, n)+(-1)^{n} \tau_{1 n \gamma(n-1) \cdots \gamma(2)}(1,2, \cdots, n)+\cdots
\end{aligned}
$$

where $+\cdots$ consists of terms $\tau_{1 \gamma(2) \cdots \gamma(n)}$ for which $\gamma(2) \neq n$ and $\gamma(n) \neq n$. Since there are only $(n-2)$ ! kinematic numerators $\mathbf{n}_{1 \gamma n}$ and $(n-1) ! / 2$ dual-trace functions $\tau_{\alpha}$, there remains a great deal of (gauge) freedom in choosing $\tau_{\alpha}$.

In refs. [31, 32], Kleiss-Kuijf relations $\sum_{\alpha} r_{\alpha} \tau_{\alpha}=0$ were imposed on $\tau_{\alpha}$, reducing the number of independent dual-trace functions to $(n-2)$ !. This is an optional ${ }^{10}$ gauge choice,

\footnotetext{
${ }^{10}$ Recall that Kleiss-Kuijf relations follow from the existence of right null vectors $\sum_{\alpha} M_{i \alpha} r_{\alpha}=0$ of the matrix $M_{i \alpha}$. By virtue of eq. (2.4), the color-ordered amplitudes $A_{\alpha}$ satisfy the relations $\sum_{\alpha} r_{\alpha} A_{\alpha}=0$. Kleiss-Kuijf relations do not apply to the trace basis $\operatorname{Tr}[\alpha]$ and therefore one is not required to impose them on $\tau_{\alpha}$. For example, see ref. [33].
} 
however, and we choose instead to set to zero all terms of the form $\tau_{1 \gamma(2) \cdots \gamma(n)}(1,2, \cdots, n)$ except

$$
\begin{aligned}
& \tau_{1 \gamma(2) \cdots \gamma(n-2) n-1, n}(1,2, \cdots, n)=-\frac{1}{2} \sum_{\beta \in S_{n-3}} S[\gamma \mid \beta] A(1, \beta(2), \cdots, \beta(n-2), n, n-1), \\
& \tau_{1, n, n-1 \gamma(n-2) \cdots \gamma(2)}(1,2, \cdots, n)=(-1)^{n} \tau_{1 \gamma(2) \cdots \gamma(n-2) n-1, n}(1,2, \cdots, n) .
\end{aligned}
$$

All remaining dual-trace functions follow from cyclic invariance (4.3). Note that our gauge choice for $\tau_{\alpha}$ also implies a particular gauge choice for $\mathbf{n}_{1 \gamma n}$, namely, eq. (3.2). According to ref. [31], the ability to express the dual-trace functions in terms of kinematic numerators requires us to impose Kleiss-Kuijf relations on $\tau_{\alpha}$. If, however, our goal is to write amplitude-encoded dual-trace functions, then this restriction is not necessary, as we will see explicitly below.

The dual-trace functions defined in eq. (4.6) are amplitude-encoded and satisfy eq. (4.2), but they are not symmetric functions in the sense of Broedel and Carrasco. ${ }^{11}$ In the appendix, it is shown that we can follow the same procedure as in the previous section to generate from eq. (4.6) a symmetric dual-trace function

$$
\tau(1,2, \cdots, n) \equiv \frac{1}{n !} \sum_{\beta \in S_{n}} \tau_{\beta^{-1}}(\beta(1), \cdots, \beta(n)) .
$$

This then provides a constructive definition for a virtuous dual-trace function for tree-level $n$-point amplitudes, proving that such a representation exists for all $n$. The symmetric dual-trace function can be used to express the $n$-gluon amplitude as

$$
\mathcal{A}(1,2, \cdots, n)=\sum_{\alpha \in S_{n} / \mathbb{Z}} \sum_{\beta \in S_{n} / \mathbb{Z}} \operatorname{Tr}[\alpha] m(\alpha \mid \beta) \tau(\beta)
$$

where $\tau(\beta) \equiv \tau(\beta(1), \beta(2), \cdots, \beta(n))$.

By substituting eq. (4.1) into (2.13), the scattering amplitude for gravitons may be written

$$
\mathcal{M}(1,2, \cdots, n)=\sum_{\alpha \in S_{n} / \mathbb{Z}} \sum_{\beta \in S_{n} / \mathbb{Z}} \tau(\alpha) m(\alpha \mid \beta) \tau(\beta) .
$$

This may equivalently be obtained by replacing $\operatorname{Tr}[\alpha]$ with $\tau_{\alpha}$ in eq. (4.8). The gravity amplitude may also be written [31]

$$
\mathcal{M}(1,2, \cdots, n)=\sum_{\alpha \in S_{n} / \mathbb{Z}} \tau(\alpha) A(\alpha)
$$

which has the nice feature of demonstrating that the gravity amplitude is manifestly invariant under an arbitrary permutation $\beta \in S_{n}$ :

$$
\mathcal{M}(\beta)=\sum_{\alpha \in S_{n} / \mathbb{Z}} \tau(\beta \alpha) A(\beta \alpha)=\sum_{\alpha^{\prime} \in S_{n} / \mathbb{Z}} \tau\left(\alpha^{\prime}\right) A\left(\alpha^{\prime}\right)=\mathcal{M}(\mathbb{1}) .
$$

In the following subsections, we compute the symmetric dual-trace function (4.7) explicitly for four- and five-point amplitudes. Despite being symmetric, our expressions do not satisfy the Kleiss-Kuijf relations, illustrating that the virtues of Broedel and Carrasco are not sufficient to single out a unique dual-trace function.

\footnotetext{
${ }^{11}$ In the language of ref. [32], they do not have a natural relabeling property.
} 


\subsection{Four-point symmetric dual-trace functions}

For the four-gluon amplitude, eq. (4.6) yields a nonsymmetric representation for the dualtrace function

$$
\begin{aligned}
& \tau_{1234}(i, j, k, l)=-\frac{1}{2} s_{i j} A(i, j, l, k), \\
& \tau_{1342}(i, j, k, l)=0 \\
& \tau_{1423}(i, j, k, l)=0
\end{aligned}
$$

with the remaining $\tau_{\alpha}$ given by eq. (4.3). We now use eq. (4.7) to generate a symmetric dual-trace function, obtaining

$$
\tau(1,2,3,4)=-\frac{1}{24}\left[\left(s_{12}+s_{34}\right) A(1,3,4,2)+\left(s_{14}+s_{23}\right) A(1,4,2,3)\right]
$$

where we have used $A(1,2,3,4)=A(4,3,2,1)$ and the cyclic invariance of $A(1,2,3,4)$. Equation (4.13) manifestly obeys $\tau(1,2,3,4)=\tau(2,3,4,1)$ and $\tau(1,2,3,4)=\tau(4,3,2,1)$; however, $\tau(1,2,3,4)+\tau(1,3,4,2)+\tau(1,4,2,3)$ does not vanish; i.e., eq. (4.13) does not satisfy the Kleiss-Kuijf relations. When substituted into eq. (4.5), which takes the form

$$
\mathbf{n}(1,2,3,4)=2[\tau(1,2,3,4)-\tau(1,2,4,3)]
$$

eq. (4.13) yields precisely the symmetric kinematic numerator found in eq. (3.8). Equation (4.13) can be written more briefly as

$$
\tau(1,2,3,4)=-\frac{1}{6} s_{12} A(1,3,4,2)
$$

by using momentum conservation and the BCJ relations (3.11).

Bern and Dennen proposed that the four-point dual-trace function takes the form [31]

$$
\tau_{B D}(1,2,3,4)=\frac{1}{6}[\mathbf{n}(1,2,3,4)+\mathbf{n}(2,3,4,1)] .
$$

The expression for $\tau$ given in ref. [32] is equivalent to eq. (4.16). Equation (4.16) manifestly obeys $\tau(1,2,3,4)=\tau(2,3,4,1)$ and $\tau(1,2,3,4)=\tau(4,3,2,1)$, and in addition satisfies the Kleiss-Kuijf relation. To compare our expression with that of Bern and Dennen, we substitute the symmetric numerator (3.8) into eq. (4.16) to find

$$
\begin{aligned}
& \tau_{B D}(1,2,3,4)-\tau(1,2,3,4) \\
& \quad=\frac{1}{36}\left[\left(s_{12}+s_{14}\right) A(1,2,3,4)+\left(s_{12}+s_{13}\right) A(1,3,4,2)+\left(s_{13}+s_{14}\right) A(1,4,2,3)\right] .
\end{aligned}
$$

The difference is "pure gauge": it vanishes when substituted into eq. (4.14), and therefore does not contribute to $\mathbf{n}(1,2,3,4)$. Hence we see that imposing the three virtues of Broedel and Carrasco on the dual-trace function is not sufficient to determine it uniquely. 
To further elucidate the difference between eqs. (4.13) and (4.16), we rewrite eq. (4.2) as a matrix equation

$$
\left(\begin{array}{l}
A_{1234} \\
A_{1342} \\
A_{1423}
\end{array}\right)=m\left(\begin{array}{c}
\tau_{1234} \\
\tau_{1342} \\
\tau_{1423}
\end{array}\right), \quad m=-\frac{2}{s t u}\left(\begin{array}{c}
u \\
t \\
s
\end{array}\right)(u t s)
$$

where $s=s_{12}, t=s_{14}$, and $u=s_{13}$. As expected, $m$ has rank $(4-3) !=1$. It therefore does not possess a unique inverse, but rather a family of generalized inverses $m^{+}$

$$
\left(\begin{array}{c}
\tau_{1234} \\
\tau_{1342} \\
\tau_{1423}
\end{array}\right)=m^{+}\left(\begin{array}{c}
A_{1234} \\
A_{1342} \\
A_{1423}
\end{array}\right), \quad \quad m^{+} m=m .
$$

A generalized inverse must satisfy $m m^{+} m=m[42,48]$, which guarantees that the resulting $\tau_{\alpha}$ will satisfy eq. (4.18).

Different gauge choices for $\tau$ correspond to different generalized inverses. The nonsymmetric representation (4.12) corresponds to

$$
m_{\text {nonsym }}^{+}=-\frac{1}{2}\left(\begin{array}{lll}
0 & s & 0 \\
0 & 0 & 0 \\
0 & 0 & 0
\end{array}\right)
$$

The symmetric dual-trace function (4.13) corresponds to

$$
m_{\mathrm{sym}}^{+}=-\frac{1}{12}\left(\begin{array}{ccc}
0 & s & t \\
s & 0 & u \\
t & u & 0
\end{array}\right)
$$

whereas the Bern-Dennen dual-trace function corresponds to

$$
m_{B D}^{+}=\frac{1}{36}\left(\begin{array}{ccc}
-u & -3 s-t & -3 t-s \\
-3 s-u & -t & -3 u-s \\
-3 t-u & -3 u-t u & -s
\end{array}\right)
$$

All three generalized inverses satisfy $m m^{+} m=m$.

\subsection{Five-point symmetric dual-trace functions}

For the five-gluon amplitude, eq. (4.6) yields a nonsymmetric representation

$$
\begin{aligned}
& \tau_{12345}(i, j, k, l, m)=-\frac{1}{2}\left[s_{i j}\left(s_{i k}+s_{j k}\right) A(i, j, k, m, l)+s_{i j} s_{i k} A(i, k, j, m, l)\right], \\
& \tau_{13245}(i, j, k, l, m)=-\frac{1}{2}\left[s_{i j} s_{i k} A(i, j, k, m, l)+s_{i k}\left(s_{i j}+s_{j k}\right) A(i, k, j, m, l)\right]
\end{aligned}
$$


with all remaining dual-trace functions set to zero, except for those related to eq. (4.23) by eq. (4.3). We now use eq. (4.7) to generate a symmetric dual-trace function, obtaining

$$
\begin{aligned}
\tau(1,2,3,4,5) & \\
=\frac{1}{120} & {\left[\left(s_{23} s_{24}+s_{34} s_{24}+2 s_{23} s_{34}\right) A(1,4,3,2,5)+\left(s_{23} s_{24}+s_{12} s_{25}\right) A(1,3,4,2,5)\right.} \\
& -\left(2 s_{12} s_{15}+s_{25} s_{15}+s_{12} s_{25}\right) A(1,2,4,3,5)+\left(s_{24} s_{34}+s_{14} s_{45}\right) A(1,4,2,3,5) \\
& -\left(s_{14} s_{15}+2 s_{45} s_{15}+s_{14} s_{45}\right) A(1,3,2,4,5) \\
& +\left(s_{34} s_{35}+s_{45} s_{35}+2 s_{34} s_{45}\right) A(1,2,5,4,3) \\
& +\left(s_{12} s_{13}+s_{14} s_{15}\right) A(1,4,5,2,3)+\left(s_{15} s_{25}+s_{35} s_{45}\right) A(1,2,5,3,4) \\
& \left.+\left(s_{12} s_{13}+s_{23} s_{13}+2 s_{12} s_{23}\right) A(1,4,5,3,2)-\left(s_{13} s_{23}+s_{34} s_{35}\right) A(1,3,5,4,2)\right]
\end{aligned}
$$

where we have used $A(1,2,3,4,5)=-A(5,4,3,2,1)$ together with cyclic invariance of $A(1,2,3,4,5)$. This expression manifestly satisfies $\tau(1,2,3,4,5)=\tau(2,3,4,5,1)$ and $\tau(1,2,3,4,5)=-\tau(5,4,3,2,1)$, but not the Kleiss-Kuijf relations. It therefore differs from the expressions given in refs. [31, 32], which do satisfy the Kleiss-Kuijf relations.

Equation (4.24) yields precisely the symmetric kinematic numerator eq. (3.16) when substituted into eq. (4.25), which takes the form

$$
\mathbf{n}(1,2,3,4,5)=2[\tau(1,2,3,4,5)+\tau(1,2,5,4,3)+\tau(1,4,5,3,2)+\tau(1,3,5,4,2)] .
$$

Finally, the Kleiss-Kuijf relations for the color-ordered amplitudes can be used to rewrite eq. (4.24) as

$$
\begin{aligned}
\tau(1,2,3,4,5) & \\
=\frac{1}{120}[ & \left(s_{12} s_{13}+2 s_{12} s_{23}+2 s_{34} s_{45}+s_{35} s_{45}\right) A(1,2,3,4,5) \\
& +\left(s_{12} s_{13}+s_{14} s_{15}+s_{23} s_{24}+s_{15} s_{25}+2 s_{23} s_{34}+s_{24} s_{34}+s_{35} s_{45}\right) A(1,4,3,2,5) \\
& +\left(s_{12} s_{13}+s_{14} s_{15}+s_{23} s_{24}+s_{12} s_{25}+s_{34} s_{35}+2 s_{34} s_{45}+s_{35} s_{45}\right) A(1,3,4,2,5) \\
& +\left(s_{12} s_{13}-2 s_{12} s_{15}+2 s_{12} s_{23}-s_{12} s_{25}-s_{34} s_{35}+s_{35} s_{45}\right) A(1,2,4,3,5) \\
& +\left(s_{12} s_{13}+s_{23} s_{13}+2 s_{12} s_{23}+s_{15} s_{25}+s_{24} s_{34}+s_{14} s_{45}+s_{35} s_{45}\right) A(1,4,2,3,5) \\
& \left.+\left(s_{12} s_{13}-s_{23} s_{13}-s_{14} s_{45}-2 s_{15} s_{45}+2 s_{34} s_{45}+s_{35} s_{45}\right) A(1,3,2,4,5)\right] .
\end{aligned}
$$

\section{Discussion}

In this paper, we have offered a constructive procedure for computing virtuous kinematic numerators for $n$-point gauge-theory scattering amplitudes; that is, numerators that simultaneously satisfy color-kinematic duality, are expressed in terms of color-ordered amplitudes, and are symmetric in the sense of Broedel and Carrasco. We have presented explicit expressions for four- and five-point amplitudes, which although somewhat lengthy, have the advantage of manifestly obeying the Jacobi identities and diagram symmetries 
without having to invoke the BCJ relations for the color-ordered amplitudes. Our results are equivalent (upon using the BCJ relations) to other virtuous expressions in the literature, suggesting the possibility that the three virtues of Broedel and Carrasco are sufficient to uniquely determine the kinematic numerators, although we do not have a proof of this.

We have also applied this procedure to compute symmetric, amplitude-encoded dualtrace functions $\tau$ for $n$-point amplitudes, presenting explicit expressions for four- and fivepoint amplitudes. In this case, the results are not uniquely determined by these criteria alone. In particular, symmetric expressions for $\tau$ obtained by other authors additionally satisfy (optional) Kleiss-Kuijf relations, whereas our results do not. While it is possible that a constructive procedure could be found to generate virtuous dual-trace functions for $n$-point amplitudes that also satisfy Kleiss-Kuijf relations, it is also possible that an alternative criterion to single out a unique dual-trace function may be more useful or natural.

\section{Acknowledgments}

I am grateful to Johannes Broedel for useful correspondence. I especially wish to thank Freddy Cachazo and Ellis Yuan for crucial conversations at the outset of this project. This research was supported in part by the NSF under grant no. PHY10-67961.

\section{A Generating symmetric kinematic numerators}

In this appendix, we consider the effect of an arbitrary permutation $\beta$ on the momentum, polarization, and color of the external particles of the $n$-gluon amplitude:

$$
\mathcal{A}(\beta) \equiv \mathcal{A}(\beta(1), \beta(2), \cdots, \beta(n)) .
$$

Of course, the full bosonic amplitude is invariant under permutations: $\mathcal{A}(\beta)=\mathcal{A}(\mathbf{1})$, where 1 denotes the identity permutation. We will explore the consequences of this on the colorordered amplitudes and kinematic numerators below.

First, we rewrite the trace decomposition (2.3) of the $n$-gluon amplitude as a sum over all permutations of the external legs

$$
\mathcal{A}(1,2, \cdots, n)=\frac{1}{n} \sum_{\alpha \in S_{n}} \operatorname{Tr}[\alpha] A_{\alpha}(1,2, \cdots, n)
$$

dividing by $n$ to correct for the overcounting. Since $\operatorname{Tr}[\alpha]=\operatorname{Tr}\left[\alpha^{\prime}\right]$ when $\alpha$ and $\alpha^{\prime}$ are related by cyclic permutations, we impose $A_{\alpha}(1,2, \cdots, n)=A_{\alpha^{\prime}}(1,2, \cdots, n)$ on the colorordered amplitudes without loss of generality. The effect of the permutation $\beta$ on the trace decomposition (A.2) is

$$
\mathcal{A}(\beta)=\frac{1}{n} \sum_{\alpha \in S_{n}} \operatorname{Tr}[\beta \alpha] A_{\alpha}(\beta)=\frac{1}{n} \sum_{\alpha^{\prime} \in S_{n}} \operatorname{Tr}\left[\alpha^{\prime}\right] A_{\beta^{-1} \alpha^{\prime}}(\beta)
$$

where $\beta \alpha$ denotes the permutation ${ }^{12}$ obtained by acting first with $\alpha$ and then with $\beta$. Since the amplitude $\mathcal{A}(1,2, \cdots, n)$ is invariant under permutations of the external legs, it follows

\footnotetext{
${ }^{12}$ Hence if $\alpha=(12)$ and $\beta=(23)$ then $\beta \alpha=(132)$, where we use the cycle notation for permutations.
} 
that

$$
\sum_{\alpha \in S_{n}} \operatorname{Tr}[\alpha] A_{\beta^{-1} \alpha}(\beta)=\sum_{\alpha \in S_{n}} \operatorname{Tr}[\alpha] A_{\alpha}(\mathbb{1}) .
$$

Since the trace basis is independent (modulo cyclic permutations) we may conclude that the color-ordered amplitudes obey

$$
A_{\beta^{-1} \alpha}(\beta)=A_{\alpha}(\mathbb{1}) \quad \Rightarrow \quad A_{\alpha}(\mathbb{1})=A_{1}(\alpha) .
$$

Denoting $A_{1}(1,2, \cdots, n)$ as simply $A(1,2, \cdots, n)$, we see that the entire set of color-ordered amplitudes $A_{\alpha}(1,2, \cdots, n)$ is obtained by permuting the arguments of this one function

$$
A_{\alpha}(1,2, \cdots, n)=A(\alpha(1), \cdots, \alpha(n))
$$

Thus, color-ordered amplitudes are symmetric in the sense of Broedel and Carrasco [29].

Next, we consider the effect of permuting the arguments of the amplitude in the color basis. We begin by rewriting the $n$-gluon amplitude eq. (2.1) as

$$
\mathcal{A}(1,2, \cdots, n)=\sum_{T} \frac{1}{\left|G^{(T)}\right|} \sum_{\alpha \in S_{n}} \frac{c_{\alpha}^{(T)} n_{\alpha}^{(T)}(1,2, \cdots, n)}{d^{(T)}(\alpha)} .
$$

Here we have divided up the color factors $c_{i}$ according to the topology $T$ of the diagram, and arbitrarily assign the color labels $\mathrm{a}_{j}$ to the legs of the diagram, calling the result $c_{1}^{(T)}$. Then $c_{\alpha}^{(T)}$ denotes the color factor with the same topology but with the color labels on the legs permuted by $\alpha \in S_{n}$. Also, $d^{(T)}(1,2, \cdots, n)$ is the denominator associated with $c_{1}^{(T)}$ and $d^{(T)}(\alpha)=d^{(T)}(\alpha(1), \cdots, \alpha(n))$.

Associated with each topology is a symmetry group $G^{(T)} \subset S_{n}$ under which the color factor is invariant (modulo sign)

$$
c_{\alpha \rho}^{(T)}= \pm c_{\alpha}^{(T)}, \quad \rho \in G^{(T)} .
$$

For example, the symmetries of the half-ladder color factor

$$
\mathbf{c}_{123 \cdots n-2, n-1, n}=-\mathbf{c}_{213 \cdots n-2, n-1, n}=-\mathbf{c}_{123 \cdots n-2, n, n-1}=(-1)^{n} \mathbf{c}_{n, n-1, n-2 \cdots 321}
$$

generate the order 8 dihedral group. In eq. (A.7), we have divided by $\left|G^{(T)}\right|$, the order of $G^{(T)}$, to correct for the overcounting that results from summing over redundant diagrams. The denominator is invariant under $G^{(T)}$

$$
d^{(T)}(\alpha)=d^{(T)}(\alpha \rho), \quad \rho \in G^{(T)}
$$

and without loss of generality, the symmetries of $c_{\alpha}^{(T)}$ may also be imposed on the numerators

$$
n_{\alpha}^{(T)}(1,2, \cdots, n)= \pm n_{\alpha \rho}^{(T)}(1,2, \cdots, n), \quad \rho \in G^{(T)}
$$

since any nonsymmetric piece will drop out of the sum over permutations. Thus we can restrict the sum to $S_{n} / G^{(T)}$

$$
\mathcal{A}(1,2, \cdots, n)=\sum_{T} \sum_{\alpha \in S_{n} / G^{(T)}} \frac{c_{\alpha}^{(T)} n_{\alpha}^{(T)}(1,2, \cdots, n)}{d^{(T)}(\alpha)} .
$$


Unlike the trace basis, the set of color factors (modulo $G^{(T)}$ ) does not constitute an independent basis on account of various Jacobi identities of the form

$$
c_{\alpha_{1}}^{\left(T_{1}\right)}+c_{\alpha_{2}}^{\left(T_{2}\right)}+c_{\alpha_{3}}^{\left(T_{3}\right)}=0
$$

where $T_{1}, T_{2}$, and $T_{3}$ need not all be equal since Jacobi identities may mix color factors with different topologies. Hence the kinematic numerators $n_{\alpha}^{(T)}$ are not unique and can undergo generalized gauge transformations.

Now we apply a permutation $\beta$ to the external legs of an amplitude expressed in the color basis (eq. (A.7)) to obtain

$$
\begin{aligned}
\mathcal{A}(\beta)=\sum_{T} \frac{1}{\left|G^{(T)}\right|} \sum_{\alpha \in S_{n}} \frac{c_{\beta \alpha}^{(T)} n_{\alpha}^{(T)}(\beta)}{d^{(T)}(\beta \alpha)} & =\sum_{T} \frac{1}{\left|G^{(T)}\right|} \sum_{\alpha^{\prime} \in S_{n}} \frac{c_{\alpha^{\prime}}^{(T)} n_{\beta^{-1} \alpha^{\prime}}^{(T)}(\beta)}{d^{(T)}\left(\alpha^{\prime}\right)} \\
& =\sum_{T} \sum_{\alpha^{\prime} \in S_{n} / G^{(T)}} \frac{c_{\alpha^{\prime}}^{(T)} n_{\beta^{-1} \alpha^{\prime}}^{(T)}(\beta)}{d^{(T)}\left(\alpha^{\prime}\right)}
\end{aligned}
$$

where in the last equation, we used

$$
n_{\beta^{-1} \alpha \rho}^{(T)}(\beta)= \pm n_{\beta^{-1} \alpha}^{(T)}(\beta), \quad \rho \in G^{(T)}
$$

which follows from eq. (A.11). The invariance of the full bosonic amplitude $\mathcal{A}(1,2, \cdots, n)$ under permutations of the external legs implies that

$$
\sum_{T} \sum_{\alpha \in S_{n} / G^{(T)}} \frac{c_{\alpha}^{(T)} n_{\beta^{-1} \alpha}^{(T)}(\beta)}{d^{(T)}(\alpha)}=\sum_{T} \sum_{\alpha \in S_{n} / G^{(T)}} \frac{c_{\alpha}^{(T)} n_{\alpha}^{(T)}(\mathbb{1})}{d^{(T)}(\alpha)}
$$

but we may not conclude that $n_{\beta^{-1} \alpha}^{(T)}(\beta)$ is equal to $n_{\alpha}^{(T)}(\mathbb{1})$, because as stated above the color factors (modulo the symmetries $G^{(T)}$ ) do not form an independent basis. In other words, the kinematic numerators need not be symmetric, in the sense of Broedel and Carrasco [29]. In particular, the kinematic numerators given in eq. (3.2) are manifestly not symmetric because some of them vanish, which is not possible if they are all related by permutations to a single function.

On the other hand, the permutation symmetry of the amplitude gives us a mechanism for generating new representations for kinematic numerators from existing ones. Specifically, eq. (A.16) demonstrates that, given a valid set of kinematic numerators $n_{\alpha}^{(T)}(\mathbb{1})$, an arbitrary permutation $\beta$ generates another valid set of numerators $n^{(T)^{\prime}}{ }_{\alpha}(\mathbf{1}) \equiv n_{\beta^{-1} \alpha}^{(T)}(\beta)$. Furthermore, if the original numerators $n_{\alpha}^{(T)}(\mathbb{1})$ are BCJ numerators, obeying

$$
n_{\alpha_{1}}^{\left(T_{1}\right)}(\mathbb{1})+n_{\alpha_{2}}^{\left(T_{2}\right)}(\mathbb{1})+n_{\alpha_{3}}^{\left(T_{3}\right)}(\mathbb{1})=0
$$

then it is also true that

$$
n_{\beta^{-1} \alpha_{1}}^{\left(T_{1}\right)}(\beta)+n_{\beta^{-1} \alpha_{2}}^{\left(T_{2}\right)}(\beta)+n_{\beta^{-1} \alpha_{3}}^{\left(T_{3}\right)}(\beta)=0
$$


which implies that the new set also obeys the Jacobi identities

$$
n_{\alpha_{1}}^{\left(T_{1}\right)^{\prime}}(\mathbf{1})+n_{\alpha_{2}}^{\left(T_{2}\right)^{\prime}}(\mathbf{1})+n_{\alpha_{3}}^{\left(T_{3}\right)^{\prime}}(\mathbf{1})=0
$$

and hence are BCJ numerators.

Moreover, normalized linear combinations of valid numerators are also valid numerators. This suggests that, given a set of nonsymmetric kinematic numerators $n_{\alpha}^{(T)}(\mathbb{1})$, one may generate a set of symmetric numerators $n_{\alpha}^{\mathrm{sym}(T)}(\mathbb{1})$ by simply averaging over all permutations

$$
n_{\alpha}^{\mathrm{sym}(T)}(\mathbf{1}) \equiv \frac{1}{n !} \sum_{\beta \in S_{n}} n_{\beta^{-1} \alpha}^{(T)}(\beta)
$$

To show that these numerators are indeed symmetric, observe that

$$
n_{\alpha}^{\operatorname{sym}(T)}(\gamma)=\frac{1}{n !} \sum_{\beta \in S_{n}} n_{\beta^{-1} \alpha}^{(T)}(\gamma \beta)=\frac{1}{n !} \sum_{\beta^{\prime} \in S_{n}} n_{\beta^{\prime-1} \gamma \alpha}^{(T)}\left(\beta^{\prime}\right)=n_{\gamma \alpha}^{\mathrm{sym}(T)}(\mathbb{1})
$$

in other words

$$
n_{\alpha}^{\operatorname{sym}(T)}(\mathbb{1})=n_{1}^{\operatorname{sym}(T)}(\alpha)
$$

Thus, all of the numerators of a given topology may be obtained by permuting the arguments of one of them. To reflect this, we drop the subscript (and the label sym) and simply define

$$
n^{(T)}(\mathbf{1}) \equiv n_{1}^{\operatorname{sym}(T)}(\mathbf{1})=\frac{1}{n !} \sum_{\beta \in S_{n}} n_{\beta^{-1}}^{(T)}(\beta)
$$

Thus the full amplitude can be written

$$
\mathcal{A}(1,2, \cdots, n)=\sum_{T} \sum_{\alpha \in S_{n} / G^{(T)}} \frac{c_{\alpha}^{(T)} n^{(T)}(\alpha)}{d^{(T)}(\alpha)}
$$

in terms of a single symmetric function $n^{(T)}(1,2, \cdots, n)$ for each topology. Furthermore, using eq. (2.20), each $n^{(T)}(1,2, \cdots, n)$ can be expressed in terms of a single symmetric function for the half-ladder diagram $\mathbf{n}(1,2, \cdots, n)$.

In a similar way, a symmetric dual-trace function can be generated from a nonsymmetric representation $\tau_{\alpha}(\mathbf{1})$. The gauge-theory amplitude may be written

$$
\begin{aligned}
\mathcal{A}(\mathbb{1}) & =\frac{1}{n^{2}} \sum_{\gamma \in S_{n}} \sum_{\delta \in S_{n}} \operatorname{Tr}[\gamma] m(\gamma \mid \delta) \tau_{\delta}(\mathbf{1}) \\
& =\frac{1}{n^{2}} \sum_{T} \frac{1}{\left|G^{(T)}\right|} \sum_{\alpha \in S_{n}} \sum_{\gamma \in S_{n}} \sum_{\delta \in S_{n}} \operatorname{Tr}[\gamma] \frac{M_{\alpha, \gamma}^{(T)} M_{\alpha, \delta}^{(T)}}{d^{(T)}(\alpha)} \tau_{\delta}(\mathbf{1}) .
\end{aligned}
$$


Applying a permutation $\beta$ to the external legs, this becomes

$$
\begin{aligned}
\mathcal{A}(\beta) & =\frac{1}{n^{2}} \sum_{T} \frac{1}{\left|G^{(T)}\right|} \sum_{\alpha \in S_{n}} \sum_{\gamma \in S_{n}} \sum_{\delta \in S_{n}} \operatorname{Tr}[\beta \gamma] \frac{M_{\alpha, \gamma}^{(T)} M_{\alpha, \delta}^{(T)}}{d^{(T)}(\beta \alpha)} \tau_{\delta}(\beta) . \\
& =\frac{1}{n^{2}} \sum_{T} \frac{1}{\mid G^{(T) \mid}} \sum_{\alpha^{\prime} \in S_{n}} \sum_{\gamma^{\prime} \in S_{n}} \sum_{\delta^{\prime} \in S_{n}} \operatorname{Tr}\left[\gamma^{\prime}\right] \frac{M_{\alpha^{\prime}, \gamma^{\prime}}^{(T)} M_{\alpha^{\prime}, \delta^{\prime}}^{(T)}}{d^{(T)}\left(\alpha^{\prime}\right)} \tau_{\beta^{-1} \delta^{\prime}}(\beta) \\
& =\frac{1}{n^{2}} \sum_{\gamma \in S_{n}} \sum_{\delta \in S_{n}} \operatorname{Tr}[\gamma] m(\gamma \mid \delta) \tau_{\beta^{-1} \delta}(\beta)
\end{aligned}
$$

where in the second line we used $M_{\alpha, \gamma}^{(T)}=M_{\beta \alpha, \beta \gamma}^{(T)}$. Thus $\tau_{\delta}(\mathbf{1})$ and $\tau_{\delta}^{\prime}(\mathbf{1}) \equiv \tau_{\beta^{-1} \delta}(\beta)$ yield the same gauge-theory amplitude. We may generate a symmetric dual-trace function $\tau(1,2, \cdots, n)$ by averaging over all permutations $\beta \in S_{n}$ :

$$
\tau(\delta(1), \cdots, \delta(n))=\tau_{\delta}^{\mathrm{sym}}(\mathbf{1}) \equiv \frac{1}{n !} \sum_{\beta \in S_{n}} \tau_{\beta^{-1} \delta}(\beta(1), \cdots, \beta(n)) .
$$

Open Access. This article is distributed under the terms of the Creative Commons Attribution License (CC-BY 4.0), which permits any use, distribution and reproduction in any medium, provided the original author(s) and source are credited.

\section{References}

[1] F. Cachazo, S. He and E.Y. Yuan, Scattering in three dimensions from rational maps, JHEP 10 (2013) 141 [arXiv: 1306.2962] [INSPIRE].

[2] F. Cachazo, S. He and E.Y. Yuan, Scattering equations and KLT orthogonality, arXiv:1306.6575 [INSPIRE].

[3] F. Cachazo, S. He and E.Y. Yuan, Scattering of massless particles in arbitrary dimension, arXiv:1307.2199 [INSPIRE].

[4] F. Cachazo, S. He and E.Y. Yuan, Scattering of massless particles: scalars, gluons and gravitons, JHEP 07 (2014) 033 [arXiv: 1309.0885] [INSPIRE].

[5] Z. Bern, J.J.M. Carrasco and H. Johansson, New relations for gauge-theory amplitudes, Phys. Rev. D 78 (2008) 085011 [arXiv: 0805.3993] [inSPIRE].

[6] Z. Bern, J.J.M. Carrasco and H. Johansson, Perturbative quantum gravity as a double copy of gauge theory, Phys. Rev. Lett. 105 (2010) 061602 [arXiv:1004.0476] [INSPIRE].

[7] S. Litsey and J. Stankowicz, Kinematic numerators and a double-copy formula for $N=4$ super-Yang-Mills residues, Phys. Rev. D 90 (2014) 025013 [arXiv:1309.7681] [INSPIRE].

[8] T. Adamo, Worldsheet factorization for twistor-strings, JHEP 04 (2014) 080 [arXiv:1310.8602] [INSPIRE].

[9] R. Monteiro and D. O'Connell, The kinematic algebras from the scattering equations, JHEP 03 (2014) 110 [arXiv:1311.1151] [INSPIRE].

[10] L. Mason and D. Skinner, Ambitwistor strings and the scattering equations, JHEP 07 (2014) 048 [arXiv:1311.2564] [INSPIRE]. 
[11] M. Chiodaroli, Q. Jin and R. Roiban, Color/kinematics duality for general abelian orbifolds of $N=4$ super Yang-Mills theory, JHEP 01 (2014) 152 [arXiv:1311.3600] [INSPIRE].

[12] L. Dolan and P. Goddard, Proof of the formula of Cachazo, He and Yuan for Yang-Mills tree amplitudes in arbitrary dimension, JHEP 05 (2014) 010 [arXiv:1311.5200] [INSPIRE].

[13] T. Adamo, E. Casali and D. Skinner, Ambitwistor strings and the scattering equations at one loop, JHEP 04 (2014) 104 [arXiv:1312.3828] [INSPIRE].

[14] H. Gomez and E.Y. Yuan, N-point tree-level scattering amplitude in the new Berkovits" string, JHEP 04 (2014) 046 [arXiv: 1312.5485] [INSPIRE].

[15] C. Kalousios, Massless scattering at special kinematics as Jacobi polynomials, J. Phys. A 47 (2014) 215402 [arXiv:1312.7743] [INSPIRE].

[16] S. Stieberger and T.R. Taylor, Closed string amplitudes as single-valued open string amplitudes, Nucl. Phys. B 881 (2014) 269 [arXiv:1401.1218] [INSPIRE].

[17] E.Y. Yuan, $\alpha^{\prime}$-expansion of open string disk integrals via Mellin transformations, arXiv: 1402.1066 [INSPIRE].

[18] S. Weinzierl, On the solutions of the scattering equations, JHEP 04 (2014) 092 [arXiv: 1402.2516] [INSPIRE].

[19] L. Dolan and P. Goddard, The polynomial form of the scattering equations, JHEP 07 (2014) 029 [arXiv: 1402.7374] [INSPIRE].

[20] N.E.J. Bjerrum-Bohr, P.H. Damgaard, P. Tourkine and P. Vanhove, Scattering equations and string theory amplitudes, arXiv:1403.4553 [INSPIRE].

[21] Y.-H. He, C. Matti and C. Sun, The scattering variety, arXiv:1403.6833 [INSPIRE].

[22] B. Kol and R. Shir, Color structures and permutations, arXiv:1403.6837 [INSPIRE].

[23] B. Kol and R. Shir, Perturbative gauge theory and $2+2=4$, arXiv:1404.1064 [INSPIRE].

[24] Y. Geyer, A.E. Lipstein and L.J. Mason, Ambitwistor strings in 4-dimensions, arXiv: 1404.6219 [INSPIRE].

[25] B.U.W. Schwab and A. Volovich, Subleading soft theorem in arbitrary dimension from scattering equations, arXiv: 1404.7749 [INSPIRE].

[26] M. Kiermaier, Gravity as the square of gauge theory, talk given at Amplitudes 2010, May 4-7, Queen Mary University of London, London, U.K. (2010).

[27] N.E.J. Bjerrum-Bohr, P.H. Damgaard, T. Sondergaard and P. Vanhove, The momentum kernel of gauge and gravity theories, JHEP 01 (2011) 001 [arXiv:1010.3933] [INSPIRE].

[28] C.R. Mafra, O. Schlotterer and S. Stieberger, Explicit BCJ numerators from pure spinors, JHEP 07 (2011) 092 [arXiv: 1104.5224] [INSPIRE].

[29] J. Broedel and J.J.M. Carrasco, Virtuous trees at five and six points for Yang-Mills and gravity, Phys. Rev. D 84 (2011) 085009 [arXiv:1107.4802] [INSPIRE].

[30] C.-H. Fu, Y.-J. Du and B. Feng, Note on symmetric BCJ numerator, arXiv:1403.6262 [INSPIRE].

[31] Z. Bern and T. Dennen, A color dual form for gauge-theory amplitudes, Phys. Rev. Lett. 107 (2011) 081601 [arXiv:1103.0312] [INSPIRE]. 
[32] Y.-J. Du, B. Feng and C.-H. Fu, The construction of dual-trace factor in Yang-Mills theory, JHEP 07 (2013) 057 [arXiv: 1304.2978] [INSPIRE].

[33] C.-H. Fu, Y.-J. Du and B. Feng, Note on construction of dual-trace factor in Yang-Mills theory, JHEP 10 (2013) 069 [arXiv: 1305.2996] [INSPIRE].

[34] S.G. Naculich, All-loop group-theory constraints for color-ordered SU(N) gauge-theory amplitudes, Phys. Lett. B 707 (2012) 191 [arXiv:1110.1859] [INSPIRE].

[35] A.C. Edison and S.G. Naculich, SU(N) group-theory constraints on color-ordered five-point amplitudes at all loop orders, Nucl. Phys. B 858 (2012) 488 [arXiv:1111.3821] [InSPIRE].

[36] A.C. Edison and S.G. Naculich, Symmetric-group decomposition of SU(N) group-theory constraints on four-, five- and six-point color-ordered amplitudes, JHEP 09 (2012) 069 [arXiv: 1207.5511] [INSPIRE].

[37] R. Kleiss and H. Kuijf, Multi-gluon cross-sections and five jet production at hadron colliders, Nucl. Phys. B 312 (1989) 616 [inSPIRE].

[38] Z. Bern, T. Dennen, Y.-t. Huang and M. Kiermaier, Gravity as the square of gauge theory, Phys. Rev. D 82 (2010) 065003 [arXiv: 1004.0693] [InSPIRE].

[39] V. Del Duca, A. Frizzo and F. Maltoni, Factorization of tree QCD amplitudes in the high-energy limit and in the collinear limit, Nucl. Phys. B 568 (2000) 211 [hep-ph/9909464] [INSPIRE].

[40] V. Del Duca, L.J. Dixon and F. Maltoni, New color decompositions for gauge amplitudes at tree and loop level, Nucl. Phys. B 571 (2000) 51 [hep-ph/9910563] [INSPIRE].

[41] D. Vaman and Y.-P. Yao, Constraints and generalized gauge transformations on tree-level gluon and graviton amplitudes, JHEP 11 (2010) 028 [arXiv: 1007.3475] [INSPIRE].

[42] R.H. Boels and R.S. Isermann, On powercounting in perturbative quantum gravity theories through color-kinematic duality, JHEP 06 (2013) 017 [arXiv: 1212.3473] [INSPIRE].

[43] N.E.J. Bjerrum-Bohr, P.H. Damgaard, B. Feng and T. Sondergaard, Gravity and Yang-Mills amplitude relations, Phys. Rev. D 82 (2010) 107702 [arXiv: 1005.4367] [INSPIRE].

[44] N.E.J. Bjerrum-Bohr, P.H. Damgaard, B. Feng and T. Sondergaard, New identities among gauge theory amplitudes, Phys. Lett. B 691 (2010) 268 [arXiv:1006.3214] [INSPIRE].

[45] N.E.J. Bjerrum-Bohr, P.H. Damgaard, B. Feng and T. Sondergaard, Proof of gravity and Yang-Mills amplitude relations, JHEP 09 (2010) 067 [arXiv:1007.3111] [INSPIRE].

[46] H. Kawai, D.C. Lewellen and S.H.H. Tye, A relation between tree amplitudes of closed and open strings, Nucl. Phys. B 269 (1986) 1 [inSPIRE].

[47] Z. Bern, L.J. Dixon, M. Perelstein and J.S. Rozowsky, Multileg one loop gravity amplitudes from gauge theory, Nucl. Phys. B 546 (1999) 423 [hep-th/9811140] [INSPIRE].

[48] A. Ben-Israel and T.N.E. Greville, Generalized inverses: theory and applications, Wiley, New York U.S.A. (1980). 